\title{
Maximum relative excitation of a specific vibrational mode via optimum laser-pulse duration
}

\author{
Xiang Zhou, ${ }^{1,2}$ Zhibin Lin, ${ }^{2,3}$ Chenwei Jiang,,${ }^{4,2}$ Meng Gao, ${ }^{2}$ and Roland E. Allen ${ }^{2, *}$ \\ ${ }^{1}$ Department of Physics, Wuhan University, Wuhan 430072, China \\ ${ }^{2}$ Department of Physics, Texas A\&M University, College Station, Texas 77843, USA \\ ${ }^{3}$ Renewable Energy Materials Research Science and Engineering Center, and Department of Physics, Colorado School of Mines, \\ Golden, Colorado 80401, USA \\ ${ }^{4}$ Department of Applied Physics, Xi'an Jiaotong University, Xi'an 710049, China
}

(Received 26 January 2010; revised manuscript received 27 May 2010; published 31 August 2010)

\begin{abstract}
For molecules and materials responding to femtosecond-scale optical laser pulses, we predict maximum relative excitation of a Raman-active vibrational mode with period $T$ when the pulse has a full-width-at-halfmaximum duration $\tau \approx 0.42 T$. This result follows from a general analytical model, and is precisely confirmed by detailed density-functional-based dynamical simulations for $\mathrm{C}_{60}$ and a carbon nanotube, which include anharmonicity, nonlinearity, no assumptions about the polarizability tensor, and no averaging over rapid oscillations within the pulse. The mode specificity is, of course, best at low temperature and for pulses that are electronically off-resonance, and the energy deposited in any mode is proportional to the fourth power of the electric field.
\end{abstract}

DOI: $10.1103 /$ PhysRevB.82.075433

PACS number(s): 78.30.Na, 78.20.Bh, 78.47.J-, 78.67.Ch

For a quarter century there has been considerable interest in optimizing the vibrational response of molecules and materials to ultrafast laser pulses. ${ }^{1-15}$ This problem is directly relevant to the broader issue of coherent control in physical, chemical, ${ }^{16-19}$ and biological ${ }^{20-23}$ systems.

Here we consider excitation via impulsive stimulated Raman scattering and related techniques using femtosecondscale optical pulses. We find that the optimum full-width-athalf-maximum (FWHM) pulse duration for exciting a specific vibrational mode with angular frequency $2 \pi / T$ is given by $\tau \approx 0.42 T$. Our prediction results from a general analytical model, and is precisely confirmed by completely independent density-functional-based simulations for $\mathrm{C}_{60}{ }^{24-28}$ and a small carbon nanotube. ${ }^{29-31}$ Unlike the model, these simulations include anharmonic effects in the vibrations, nonlinear effects in the response to the applied field and no simplifying assumptions about the electronic polarizability tensor.

Our general model consists of the following: (1) the electric field has the form

$$
\mathcal{E}(t)=\mathcal{E}_{0} \sin (\pi t / 2 \tau) \sin (\omega t+\delta), \quad 0<t<2 \tau
$$

with $\omega \gg \pi / \tau$ and $\hbar \omega$ off resonance. Since the oscillations of $\sin ^{2}(\omega t+\delta)$ will average out to $1 / 2$ over a period that is short compared to the response time of the vibrating nuclei, we will actually replace the square of Eq. (1) by the envelope function

$$
\overline{\mathcal{E}}(t)^{2}=\overline{\mathcal{E}}_{0}^{2} \sin ^{2}(\pi t / 2 \tau), \quad 0<t<2 \tau
$$

with $\overline{\mathcal{E}}_{0}^{2}=\mathcal{E}_{0}^{2} / 2$. This form has the following nice features: ${ }^{32}$ (i) the duration is finite and need not be truncated. (ii) The FWHM duration is exactly half the full duration $2 \tau$. (iii) A plot reveals that it closely resembles a Gaussian. (iv) The slope is zero at beginning and end. (2) The initial conditions $Q_{k}(0)=\dot{Q}_{k}(0)=0$ are imposed on the normal-mode coordinates $Q_{k}(t)$. This approximation is valid for the expectation value below Eq. (4) at low temperature, or an ensemble av- erage at higher temperature, in the linearized Eq. (3). (3) The equation of motion is given by the standard (Placzek) model for Raman-active modes $8,21,33$

$$
d^{2} Q_{k} / d t^{2}+\omega_{k}^{2} Q_{k}=\alpha_{k}^{\prime} \mathcal{E}(t)^{2} / 2
$$

where the anharmonic and damping terms (in the normalmode coordinates $Q_{k}$ ), nonlinear terms [in the electric field $\mathcal{E}(t)]$, and off-diagonal terms (in the polarizability tensor $\boldsymbol{\alpha}$ ) have been neglected, with the electric dipole moment given by $\boldsymbol{\mu}_{\mathrm{tot}}=\boldsymbol{\mu}+\boldsymbol{\mu}_{\text {ind }}, \boldsymbol{\mu}_{\text {ind }}=\boldsymbol{\alpha} \cdot \mathcal{E}+\cdots, \alpha_{x x}=\alpha_{0}+\sum_{k} \alpha_{k}^{\prime} Q_{k}+\cdots$, and the radiation field taken to be linearly polarized in the $x$ direction. Equation (3) follows from, e.g., the Heisenberg equations of motion for the quantized Hamiltonian

$$
\hat{H}=\sum_{k}\left(\hat{P}_{k}^{2} / 2+\omega_{k}^{2} \hat{Q}_{k}^{2} / 2\right)-\hat{\boldsymbol{\mu}} \cdot \mathcal{E}-\mathcal{E} \cdot \hat{\boldsymbol{\alpha}} \cdot \mathcal{E} / 2
$$

with $Q_{k} \equiv\left\langle\hat{Q}_{k}\right\rangle$ and $\boldsymbol{\alpha}_{k}^{\prime} \equiv\left\langle\partial \hat{\boldsymbol{\alpha}} / \partial \hat{Q}_{k}\right\rangle$, when the term involving $\partial \hat{\boldsymbol{\mu}} / \partial Q_{k}$ vanishes or is neglected (e.g., in taking rapid oscillations of the electric field $\mathcal{E}(t)$ to average out to zero, an approximation which also causes the lowest-order nonlinear term to vanish in Eq. (4)).

If Eq. (2) is substituted into Eq. (3), the solution after the pulse is found to reduce to

$$
Q_{k}(t)=\frac{\alpha_{k}^{\prime} \mathcal{E}_{0}^{2}}{4 \omega_{k}^{2}} \frac{\sin \left(r_{k} \pi\right)}{1-r_{k}^{2}} \sin \left(\omega_{k} t-r_{k} \pi\right), \quad t \geq 2 \tau,
$$

where

$$
r_{k}=2 \tau / T_{k}
$$

The total energy $\left(\dot{Q}_{k}^{2}+\omega_{k}^{2} Q_{k}^{2}\right) / 2$ in vibrational mode $k$ is therefore

$$
E_{k}=\frac{1}{32}\left[\frac{\alpha_{k}^{\prime}}{\omega_{k}} \frac{\sin \left(r_{k} \pi\right)}{1-r_{k}^{2}}\right]^{2} \mathcal{E}_{0}^{4}, \quad t \geq 2 \tau .
$$

$E_{k}$ is, of course, equivalent to the maximum kinetic energy $K_{k}^{\max }$. 
The maximum response is then given by the extremum of the function in brackets, which occurs at $r_{k} \approx 0.8375$ or $2 \tau$ $\approx 0.8375 T_{k}$. We have tested this prediction by performing independent supercomputer simulations for $\mathrm{C}_{60}$ and a small carbon nanotube, using the density-functional-based approach of Frauenheim and co-workers, ${ }^{34,35}$ together with semiclassical electron-radiation-ion dynamics (SERID), which is defined by the following equations. ${ }^{36}$

(1) Time-dependent Schrödinger equation in a nonorthogonal basis

$$
i \hbar \partial \boldsymbol{\psi}(t) / \partial t=\boldsymbol{S}^{-1} \cdot \boldsymbol{H} \cdot \boldsymbol{\psi}(t) .
$$

With 60 atoms and a minimal basis set, the matrices are $240 \times 240$. A time step of 50 attoseconds was used. The simulation time after completion of the pulse was $2000 \mathrm{fs}$ for $\mathrm{C}_{60}$ and $1000 \mathrm{fs}$ for the nanotube.

(2) Ehrenfest's theorem (in a nonorthogonal basis)

$$
M \frac{d^{2} X}{d t^{2}}=-\frac{1}{2} \sum_{n} \boldsymbol{\psi}_{n}^{\dagger} \cdot\left(\frac{\partial \boldsymbol{H}}{\partial X}-i \hbar \frac{\partial \boldsymbol{S}}{\partial X} \frac{\partial}{\partial t}\right) \cdot \boldsymbol{\psi}_{n}+\text { h.c. }-\frac{\partial U_{r e p}}{\partial X},
$$

where $X$ is any nuclear coordinate. The Hamiltonian matrix $\boldsymbol{H}$, overlap matrix $\boldsymbol{S}$, and effective ion-ion repulsion $U_{\text {rep }}$ were determined by the methods and results of Refs. 34 and 35 and later work by this group.

(3) Coupling of electrons to the radiation field through the time-dependent Peierls substitution

$$
H\left(\ell^{\prime}, \ell\right)=H_{0}\left(\ell^{\prime}, \ell\right) e^{i q A(t) \cdot\left(X^{\prime}-X\right) / \hbar c},
$$

where $q=-e . \boldsymbol{A}$ is the vector potential, which in the present simulations was taken to have the form

$$
\boldsymbol{A}(t)=\boldsymbol{A}_{0} \sin (\pi t / 2 \tau) \cos (\omega t), \quad 0 \leq t \leq 2 \tau
$$

with $\omega \gg \pi / \tau$, so that $\mathcal{E}(t) \approx \omega A_{0} \sin (\pi t / 2 \tau) \sin (\omega t)$, although Eq. (11) was actually used. The polarization vector was taken to lie along the $x$ axis, with the $z$ axis pointing down the axis of a nanotube.

Within the present density functional theory (DFT)-based model, the energy gap for electronic excitations is $1.80 \mathrm{eV}$ for $\mathrm{C}_{60}$ and $1.56 \mathrm{eV}$ for a $(3,3)$ nanotube with a periodicity length of 5 unit cells. [This model nanotube, with only 60 atoms, has a substantial gap because small wavenumbers $k_{z}$ are not allowed. An infinitely long $(3,3)$ nanotube would be metallic. $\left.{ }^{30}\right]$ The laser pulse photon energy was chosen to be $0.69 \mathrm{eV}$ for $\mathrm{C}_{60}$ and $0.80 \mathrm{eV}$ for the nanotube, and is thus off-resonance in both cases.

For $\mathrm{C}_{60}$, seven of the ten Raman-active modes were appreciably excited: modes $A_{g}(1), A_{g}(2), H_{g}(1), H_{g}(4), H_{g}(5)$, $H_{g}(6)$, and $H_{g}(8)$, with periods of $59.7 \mathrm{fs}, 20.6 \mathrm{fs}, 125 \mathrm{fs}$, $38.5 \mathrm{fs}, 27.0 \mathrm{fs}, 23.0 \mathrm{fs}$, and $19.0 \mathrm{fs}$ respectively. For the $(3,3)$ nanotube only an $E_{g}$ mode with a period of 182 fs was observed, and this is consistent with the experimental difficulty of observing the radial breathing mode in the Raman spectrum. ${ }^{31}$

As in Ref. 12, it is natural to characterize the strength of the vibrational response of a specific mode by its maximum kinetic energy $K_{k}^{\max }$. In the harmonic approximation, after

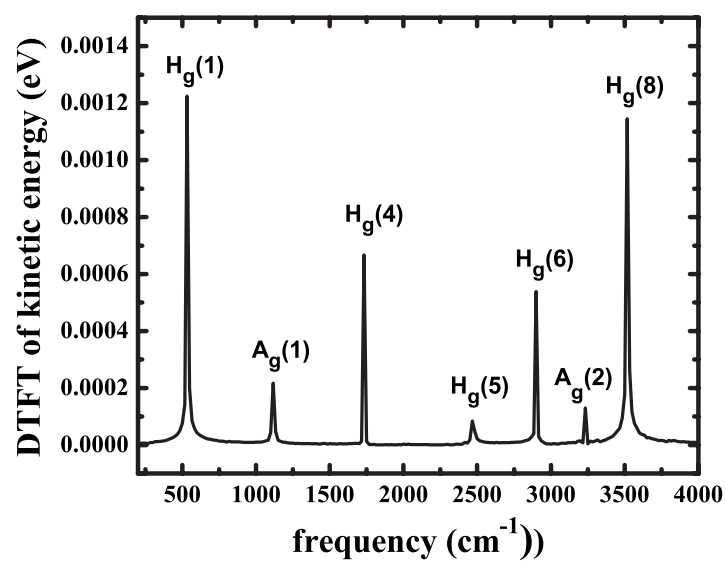

FIG. 1. Numerical Fourier transform of the total kinetic energy of $\mathrm{C}_{60}$ after being subjected to a laser pulse with a FWHM duration $\tau$ of $12 \mathrm{fs}$, photon energy $\hbar \omega$ of $0.69 \mathrm{eV}$, and electric field amplitude $\mathcal{E}_{0}$ of $5.0 \mathrm{~V} / \mathrm{nm}$. Seven Raman-active modes are clearly observed, each with a frequency $\omega_{k} / 2 \pi$ which is half the frequency exhibited in the figure by the corresponding kinetic energy $K_{k}$.

completion of the laser pulse, the velocity $\dot{Q}_{k}(t)$ is proportional to $\cos \left(\omega_{k} t+\delta\right)$, so $K_{k}$ is proportional to $\cos ^{2}\left(\omega_{k} t+\delta\right)$ $=\left[1+\cos \left(2 \omega_{k} t+2 \delta\right)\right] / 2$. A numerical Fourier transform of the total kinetic energy therefore shows a peak at $2 \omega_{k}$, with a strength proportional to the response of the normal mode with angular frequency $\omega_{k}$. Figure 1 shows our results for the Fourier transform of the total kinetic energy for $\mathrm{C}_{60}$ following a $12 \mathrm{fs}, 0.69 \mathrm{eV}, 5.0 \mathrm{~V} / \mathrm{nm}$ pulse.

Figure 2 shows that the detailed simulations agree with Eq. (7) regarding the dependence of the maximum kinetic energy $K_{k}^{\max }$ of mode $k$ on the field amplitude $\mathcal{E}_{0}$ (for the range of amplitudes considered here) when the pulse duration is fixed, $K_{k}^{\max } \propto \mathcal{E}_{0}^{4}$. When $\mathcal{E}_{0}$ is fixed, on the other hand, $K_{k}^{\max }$ is predicted to be proportional to the square of the

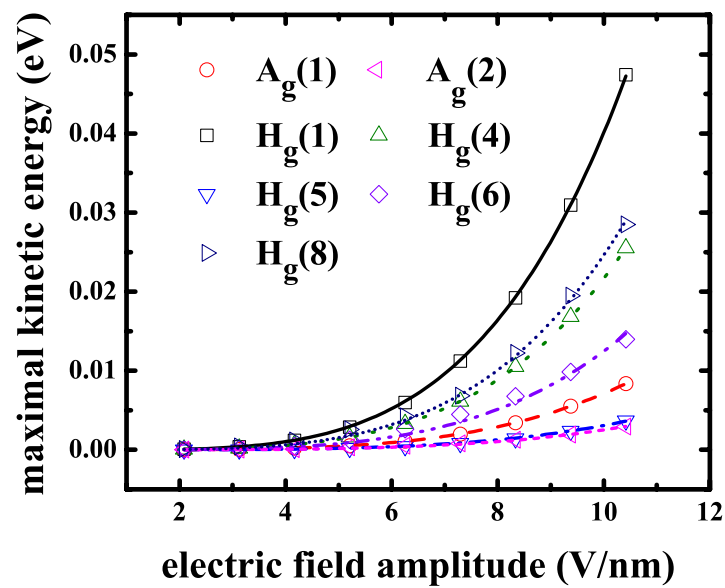

FIG. 2. (Color online) Maximum kinetic energy of $A_{g}(1), A_{g}(2)$, $H_{g}(1), H_{g}(4), H_{g}(5), H_{g}(6)$, and $H_{g}(8)$ modes of $\mathrm{C}_{60}$ responding to $0.69 \mathrm{eV}$ laser pulses when the FWHM pulse duration $\tau$ is fixed at 12 fs and the electric field amplitude $\mathcal{E}_{0}$ is varied. Here, and in the subsequent figures, each point represents a DFT-based SERID simulation and each curve has the form predicted by Eq. (7), with no adjustable parameters except the effective polarizability parameter $\alpha_{k}^{\prime}$, which is determined by a least-squares fit. 


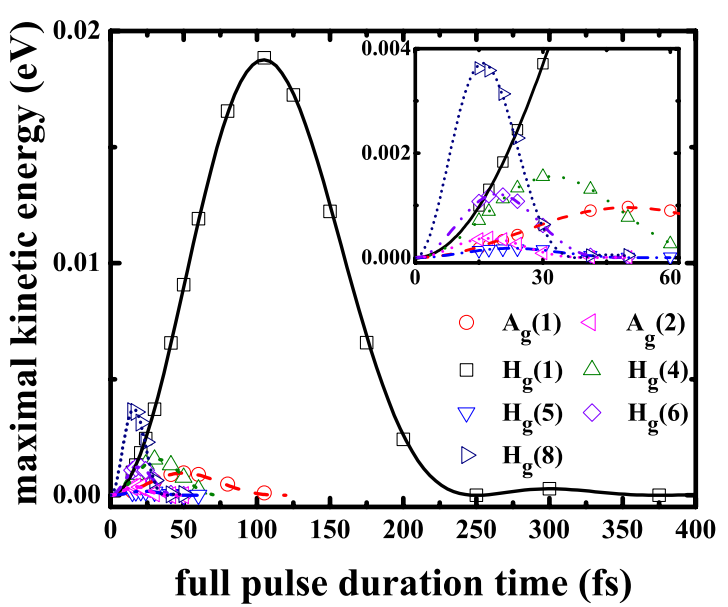

FIG. 3. (Color online) Maximum kinetic energy for the seven prominent modes of $\mathrm{C}_{60}$ responding to $0.69 \mathrm{eV}$ laser pulses when $\mathcal{E}_{0}$ is fixed at $5.0 \mathrm{~V} / \mathrm{nm}$ and the full pulse duration time $2 \tau$ is varied. The agreement between the general model of Eqs. (2) and (3) (curves) and the density-functional-based simulations of Eqs. (8)-(11) (points) is remarkable.

function involving $r_{k}$ in Eq. (7), and is maximal when $r_{k}$ $\approx 0.84$. Figure 3 shows, for example, that the $H_{g}(8)$ mode in $\mathrm{C}_{60}$ dominates for $2 \tau<23.4 \mathrm{fs}$, and the $H_{g}(1)$ mode for $2 \tau$ $>23.4$ fs, in agreement with the analytical model of Eqs. (2) and (3), and in qualitative agreement with other simulations $^{12}$ and experiment. ${ }^{24-28}$

In Fig. 3, the agreement between the predictions of the analytical model (curves) and the completely independent DFT-based simulations (points) is truly remarkable. The only adjustable parameter for each curve is the effective polarizability parameter $\alpha_{k}^{\prime}$.

There is similarly remarkable agreement for the carbon nanotube, as can be seen in Fig. 4. In this case, two photon energies $\hbar \omega$ were used: $0.8 \mathrm{eV}$ and $2.0 \mathrm{eV}$, which are respectively below and above the excitation gap of $1.56 \mathrm{eV}$ for this
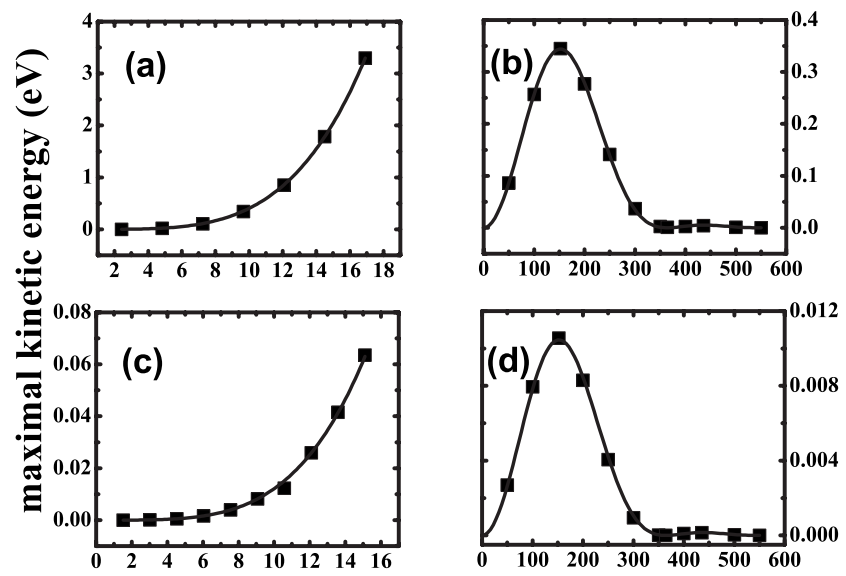

electric field amplitude (V/nm) full pulse duration time (fs)

FIG. 4. Maximum kinetic energy of $E_{g}$ mode for (3,3) carbon nanotube, excited by a $0.8 \mathrm{eV}$ laser pulse (a) when $\tau$ is fixed at 76 fs and $\mathcal{E}_{0}$ is varied and (b) when $\mathcal{E}_{0}$ is fixed at $9.66 \mathrm{~V} / \mathrm{nm}$ and $\tau$ is varied. Panels (c) and (d) are the corresponding results for a pulse with a photon energy of $2.0 \mathrm{eV}$.
TABLE I. For each of the prominent modes observed in the response of $\mathrm{C}_{60}$ and the $(3,3)$ carbon nanotube, the values of the effective $\alpha_{k}^{\prime}$ were obtained through the procedures designated (a) and (b) in the text. In procedure (a), $\mathcal{E}_{0}$ was varied while the (FWHM) duration $\tau$ was fixed at $12 \mathrm{fs}$, for $\mathrm{C}_{60}$, or $76 \mathrm{fs}$, for the nanotube. In procedure (b), $\tau$ was varied while $\mathcal{E}_{0}$ was fixed at 5.0 $\mathrm{V} / \mathrm{nm}$, for $\mathrm{C}_{60}$, or $9.66 \mathrm{~V} / \mathrm{nm}$, for the nanotube. The nanotube was also subjected to $2.0 \mathrm{eV}$ pulses, and the effective values of $\alpha_{k}^{\prime}$ obtained through both procedures are again shown.

\begin{tabular}{lccc}
\hline \hline & Mode & $\alpha_{k}^{\prime}(\mathrm{a})$ & $\alpha_{k}^{\prime}(\mathrm{b})$ \\
\hline Carbon nanotube & $E_{g}$ & $7.578 \times 10^{-4}$ & $7.496 \times 10^{-4}$ \\
$\mathrm{C}_{60}$ & $A_{g}(1)$ & $4.416 \times 10^{-4}$ & $4.505 \times 10^{-4}$ \\
& $A_{g}(2)$ & $6.248 \times 10^{-4}$ & $8.102 \times 10^{-4}$ \\
& $H_{g}(1)$ & $9.668 \times 10^{-4}$ & $9.516 \times 10^{-4}$ \\
& $H_{g}(4)$ & $8.978 \times 10^{-4}$ & $8.884 \times 10^{-4}$ \\
& $H_{g}(5)$ & $4.482 \times 10^{-4}$ & $4.294 \times 10^{-4}$ \\
& $H_{g}(6)$ & $1.127 \times 10^{-3}$ & $1.316 \times 10^{-3}$ \\
& $H_{g}(8)$ & $2.376 \times 10^{-3}$ & $2.788 \times 10^{-3}$ \\
& $E_{g}$ & $1.314 \times 10^{-4}$ & $1.307 \times 10^{-4}$ \\
\hline \hline
\end{tabular}

model nanotube, as discussed above. Figure 4 shows the results for the maximum kinetic energy $K_{k}^{\max }$ of the one prominent mode observed in this case. (Recall that the polarization vector for the pulse was chosen to point across the nanotube, so that only radial modes should be directly excited and this is a severe constraint for the small $(3,3)$ nanotube, as is also observed in experiment. ${ }^{31}$ ) The panels in Fig. 4 correspond to: (a) $\hbar \omega=0.8 \mathrm{eV}, \quad \tau=76 \mathrm{fs} ; \quad$ (b) $\hbar \omega=0.8 \mathrm{eV}, \quad \mathcal{E}_{0}$ $=9.66 \mathrm{~V} / \mathrm{nm} ;$ (c) $\hbar \omega=2.0 \mathrm{eV}, \quad \tau=76 \mathrm{fs} ;$ and (d) $\hbar \omega$ $=2.0 \mathrm{eV}, \mathcal{E}_{0}=9.66 \mathrm{~V} / \mathrm{nm}$. It is interesting that the analytical model still works in this case even for above-the-gap excitation, but we find less satisfactory agreement for $\mathrm{C}_{60}$, and certainly do not expect the model of Eq. (3) to be generally valid when there is a substantial population of electrons in excited states at the end of the pulse. In this case other effects will ordinarily be of dominant importance. ${ }^{4,11}$

As mentioned above, the electronic response in the DFTbased simulations can be characterized by an effective polarizability parameter $\alpha_{k}^{\prime}$ if one fits Eq. (7) to the results of the simulations. If the model leading to Eq. (7) is in fact consistent with the detailed simulations, then the values obtained when (a) varying the electric field amplitude $\mathcal{E}_{0}$ at constant (FWHM) pulse duration $\tau$ and (b) varying $\tau$ at constant $\mathcal{E}_{0}$ should also be reasonably consistent. Table I shows the results for all of the prominent Raman-active modes. The two procedures do lead to generally consistent values, with the differences for the $A_{g}(2), H_{g}(6)$, and $H_{g}(8)$ modes presumably arising from their short periods, so that the approximation of averaging out the $\omega$ oscillations in Eq. (2) is not valid. However, the prediction regarding optimum pulse duration works even for these modes, as can be seen in Fig. 3 .

It has long been suspected that there might be an optimum pulse duration. For example, in Ref. 12 the pulse envelope was assumed to have a Gaussian form: $|\mathcal{E}(t)|^{2}=$ $A \exp \left(-2 t^{2} / \bar{\tau}^{2}\right) \cos ^{2}(\omega t)$ (with a slight change in notation). Simulations were then performed with a model that essen- 
tially lies between the analytical model of Eqs. (2) and (3) and our much more extensive density-functional-based simulations, defined by Eqs. (8)-(11). It was found that the optimum value of $\bar{\tau}$ was about $T / 3.4$. The corresponding FWHM duration then has an optimum value $\tau=\sqrt{2 \ln 2} \bar{\tau} \approx 0.35 T$. In both our general model and DFT-based simulations, on the other hand, the optimal FWHM pulse duration is found to be given by $\tau \approx 0.42 T$. Both results seem to be consistent with the existing experiments, ${ }^{24-28}$ and both are larger than the naïve prediction of $\tau=0.25 T$. In our result, the reason appears to be the phase lag apparent in Eq. (5).

It should be emphasized that our result of $\tau \approx 0.42 T$ is for the maximum response of this mode when the intensity of the laser pulse is kept fixed while the duration is varied. On the other hand, if the fluence is instead held fixed (while $\tau$ is varied), Eqs. (5) and (7) are changed to

$$
Q_{k}(t)=\frac{\alpha_{k}^{\prime} W}{\omega_{k} \pi c \epsilon_{0}} \frac{\sin \left(r_{k} \pi\right)}{r_{k}\left(1-r_{k}^{2}\right)} \sin \left(\omega_{k} t-r_{k} \pi\right), \quad t \geq 2 \tau
$$

and

$$
E_{k}=\frac{1}{2}\left[\frac{\alpha_{k}^{\prime} W}{\pi c \epsilon_{0}} \frac{\sin \left(r_{k} \pi\right)}{r_{k}\left(1-r_{k}^{2}\right)}\right]^{2}, \quad t \geq 2 \tau
$$

where

$$
W=\frac{c \epsilon_{0}}{4} \mathcal{E}_{0}^{2} \tau
$$

is the energy in the pulse. If $W$ is held constant, the maximum in Eq. (13) is achieved as $r_{k} \rightarrow 0$

$$
E_{k}(0)=\frac{1}{2}\left[\frac{\alpha_{k}^{\prime} W}{c \epsilon_{0}}\right]^{2}
$$

so that

$$
\frac{E_{k}(\tau)}{E_{k}(0)}=\left[\frac{\sin \left(r_{k} \pi\right)}{\pi r_{k}\left(1-r_{k}^{2}\right)}\right]^{2}
$$

Then $r_{k}=0.84$ gives $E_{k}(0.42 T) / E_{k}(0)=0.385$. However, Eq.
(15) holds for all modes, so there is no relative enhancement of any preferred mode.

It should also be emphasized that the success of the simple analytical model of Eq. (3) results from the fact that the additional effects described below Eq. (3) are not very important for the pulse intensities, time durations, and systems considered here. The various effects omitted in the model will lead to richer (or messier) behavior in regimes where nonlinear effects, anharmonicity, damping, etc., become comparable in importance to the lowest-order effects included in the model. In future studies with densityfunctional-based simulations based on Eqs. (8)-(11) it may be interesting to sort out the influence of the higher-order effects.

Finally, as emphasized below Eq. (1), the present results are for $\hbar \omega$ off resonance, in contrast to those of, e.g., Refs. 11 and 14. In particular, Smith and Cina considered pulses whose central frequencies are near resonance with an electronic transition, in a model with two electronic levels and one vibrational degree of freedom. They found that the momentum increment (for the nuclei) falls off less rapidly with increasing offset (from resonance) than does the population loss (from the electronic ground state), and they presented a rather complex strategy for optimizing the various parameters for these "preresonant" pulses. The work of this group and others is thus complementary to that of the present paper.

In summary, we find remarkable agreement between the general model of Eqs. (2) and (3) and the detailed DFT-based simulations based on Eqs. (8)-(11) for $\mathrm{C}_{60}$ and a carbon nanotube. At fixed pulse intensity, both of these approaches predict maximum excitation of a Raman-active vibrational mode with period $T$ when the pulse has an FWHM duration $\tau \approx 0.42 T$.

This work was supported by the Robert A. Welch Foundation (Grant No. A-0929) and the China Scholarship Council, and we wish to thank the Texas A\&M University Supercomputing Facility for the use of its parallel computing resources.

\footnotetext{
*allen@tamu.edu

${ }^{1}$ S. De Silvestri, J. G. Fujimoto, E. P. Ippen, E. B. Gamble, Jr., L. R. Williams, and K. A. Nelson, Chem. Phys. Lett. 116, 146 (1985).

${ }^{2}$ Y.-X. Yan, E. B. Gamble, Jr., and K. A. Nelson, J. Chem. Phys. 83, 5391 (1985).

${ }^{3}$ A. M. Weiner, D. E. Leaird, G. P. Wiederrecht, and K. A. Nelson, Science 247, 1317 (1990).

${ }^{4}$ H. J. Zeiger, J. Vidal, T. K. Cheng, E. P. Ippen, G. Dresselhaus, and M. S. Dresselhaus, Phys. Rev. B 45, 768 (1992).

${ }^{5}$ C. J. Bardeen, Q. Wang, and C. V. Shank, Phys. Rev. Lett. 75, 3410 (1995).

${ }^{6}$ A. Nazarkin and G. Korn, Phys. Rev. A 58, R61 (1998).

${ }^{7}$ A. Nazarkin, G. Korn, M. Wittmann, and T. Elsaesser, Phys. Rev. Lett. 83, 2560 (1999).

${ }^{8}$ R. A. Bartels, S. Backus, M. M. Murnane, and H. C. Kapteyn,
}

Chem. Phys. Lett. 374, 326 (2003).

${ }^{9}$ S.-Y. Lee, D. Zhang, D. W. McCamant, P. Kukura, and R. A. Mathies, J. Chem. Phys. 121, 3632 (2004).

${ }^{10}$ H. Niikura, D. M. Villeneuve, and P. B. Corkum, Phys. Rev. Lett. 92, 133002 (2004).

${ }^{11}$ B. Torralva, T. A. Niehaus, M. Elstner, S. Suhai, Th. Frauenheim, and R. E. Allen, Phys. Rev. B 64, 153105 (2001).

${ }^{12}$ G. P. Zhang and T. F. George, Phys. Rev. Lett. 93, 147401 (2004); Phys. Rev. B 73, 035422 (2006).

${ }^{13}$ U. Banin, A. Bartana, S. Ruhman, and R. Kosloff, J. Chem. Phys. 101, 8461 (1994).

${ }^{14}$ T. J. Smith and J. A. Cina, J. Chem. Phys. 104, 1272 (1996).

${ }^{15}$ T. E. Stevens, J. Hebling, J. Kuhl, and R. Merlin, Phys. Status Solidi B 215, 81 (1999).

${ }^{16}$ H. Rabitz, R. de Vivie-Riedle, M. Motzkus, and K. Kompa, Science 288, 824 (2000). 
${ }^{17}$ T. C. Weinacht, R. Bartels, S. Backus, P. H. Bucksbaum, B. Pearson, J. M. Geremia, H. Rabitz, H. C. Kapteyn, and M. M. Murnane, Chem. Phys. Lett. 344, 333 (2001).

${ }^{18}$ R. A. Bartels, T. C. Weinacht, S. R. Leone, H. C. Kapteyn, and M. M. Murnane, Phys. Rev. Lett. 88, 033001 (2002).

${ }^{19}$ A. Assion, T. Baumert, M. Bergt, T. Brixner, B. Kiefer, V. Seyfried, M. Strehle, and G. Gerber, Science 282, 919 (1998).

${ }^{20}$ K. T. Tsen, S.-W. D. Tsen, C.-L. Chang, C.-F. Hung, T. C. Wu, and J. G. Kiang, Virol J. 4, 50 (2007).

${ }^{21}$ K. T. Tsen, S.-W. D. Tsen, O. F. Sankey, and J. G. Kiang, J. Phys.: Condens. Matter 19, 472201 (2007).

${ }^{22}$ E. C. Dykeman and O. F. Sankey, Phys. Rev. Lett. 100, 028101 (2008).

${ }^{23}$ E. C. Dykeman and O. F. Sankey, J. Phys.: Condens. Matter 21, 505102 (2009).

${ }^{24}$ S. L. Dexheimer, D. M. Mittleman, R. W. Schoenlein, W. Vareka, X.-D. Xiang, A. Zettl, and C. V. Shank, in Ultrafast Phenomena VIII, edited by J. L. Martin, A. Migus, G. A. Mourou, and A. H. Zewail (Springer-Verlag, Berlin, 1993), p. 81.

${ }^{25}$ H. Hohmann, C. Callegari, S. Furrer, D. Grosenick, E. E. B. Campbell, and I. V. Hertel, Phys. Rev. Lett. 73, 1919 (1994).
${ }^{26}$ M. Boyle, T. Laarmann, K. Hoffmann, M. Hedén, E. E. B. Campbell, C. P. Schulz, and I. V. Hertel, Eur. Phys. J. D 36, 339 (2005).

${ }^{27}$ T. Laarmann, I. Shchatsinin, A. Stalmashonak, M. Boyle, N. Zhavoronkov, J. Handt, R. Schmidt, C. P. Schulz, and I. V. Hertel, Phys. Rev. Lett. 98, 058302 (2007).

${ }^{28}$ V. R. Bhardwaj, P. B. Corkum, and D. M. Rayner, Phys. Rev. Lett. 91, 203004 (2003).

${ }^{29}$ Carbon Nanotubes, Advanced Topics in the Synthesis, Structure, Properties and Applications, edited by A. Jorio, G. Dresselhaus, and M. S. Dresselhaus (Springer, Berlin, 2008).

${ }^{30}$ H. J. Liu and C. T. Chan, Phys. Rev. B 66, 115416 (2002).

${ }^{31}$ M. Hulman, H. Kuzmany, O. Dubay, G. Kresse, L. Li, Z. K. Tang, P. Knoll, and R. Kaindl, Carbon 42, 1071 (2004).

${ }^{32}$ J. S. Graves and R. E. Allen, Phys. Rev. B 58, 13627 (1998).

${ }^{33}$ Y. R. Shen and N. Bloembergen, Phys. Rev. 137, A1787 (1965).

${ }^{34}$ D. Porezag, Th. Frauenheim, Th. Köhler, G. Seifert, and R. Kaschner, Phys. Rev. B 51, 12947 (1995).

${ }^{35}$ G. Seifert, D. Porezag, and Th. Frauenheim, Int. J. Quantum Chem. 58, 185 (1996).

${ }^{36}$ R. E. Allen, Phys. Rev. B 78, 064305 (2008), and references therein. 